\title{
Relationship between Pathological Characteristics and Radiological Findings on Perfusion MR Imaging of Meningioma
}

\author{
Atsushi SAITO, ${ }^{1}$ Takashi InOUE, ${ }^{2}$ Shinsuke SuZUKI, ${ }^{2}$ Masayuki EZURA, ${ }^{2}$ \\ Hiroshi UENOHARA, ${ }^{2}$ and Teiji TOMINAGA ${ }^{3}$ \\ ${ }^{1}$ Department of Neurosurgery, Kohnan Hospital, Sendai, Miyagi, Japan \\ ${ }^{2}$ Department of Neurosurgery, Sendai Medical Center, Sendai, Miyagi, Japan \\ ${ }^{3}$ Department of Neurosurgery, Tohoku University Graduate School of Medicine, \\ Sendai, Miyagi, Japan
}

\begin{abstract}
Few studies have reviewed the roles of perfusion magnetic resonance (MR) imaging in the histopathological examination of meningiomas. We analyzed the relationships between radiological findings on perfusion MR imaging and pathological characteristics such as origin of the tumor, mitotic activity, pathological subtype, and perifocal edema formation. The subjects were 21 surgical cases of meningioma preoperatively evaluated by perfusion MR imaging. A region of interest (ROI) was set inside of the tumor, and perifocal edema of the same size, cerebral blood volume (CBV), and cerebral blood flow (CBF) on perfusion MR and diffusion-weighted (DW) imaging were analyzed. These radiological data were evaluated in comparison with histopathological characteristics. On perfusion MR imaging, the average ratio of CBV against the contralateral side was $6.43(1.13-20.0)$ and that of $C B F$ was $7.73(1.34-11.3)$. There was no significant relationship with perfusion MR imaging data, tumor volume, or perifocal edema volume. However, the large peritumoral edema group often had a higher CBV and CBF than the non-large peritumoral edema group. The skull base group had a significantly higher CBV and lower signal intensity on DW images than the non-skull base group. Signal intensity on DW images was higher in grade II or III than in grade I. Perfusion MR imaging data revealed that the higher ratio of peritumoral edema against tumor size was associated with higher blood flow and blood volume under intratumoral circulatory conditions, and that skull base meningioma had a higher blood volume than non-skull base meningioma.
\end{abstract}

Keywords: meningioma, perfusion, magnetic resonance, circulation

\section{Introduction}

Meningiomas account for $30 \%$ of primary brain tumors. ${ }^{1,2)}$ Several magnetic resonance (MR) imaging techniques have been demonstrated to provide vascular information of meningiomas that was previously only available by conventional angiography. ${ }^{1)}$ Arterial spin labeling and regional perfusion imaging techniques can confirm whether the vascular

Received April 28, 2020; Accepted November 6, 2020

Copyright@ 2021 by The Japan Neurosurgical Society This work is licensed under a Creative Commons AttributionNonCommercial-NoDerivatives International License. supply of a meningioma is from the external carotid artery, the internal carotid artery, or both. ${ }^{1,3)}$ Perfusion imaging is an MR technique primarily applied to the categorization of stroke, but it can also provide useful information about neoplasms. ${ }^{4,5}$ Both diffusion tensor imaging and perfusion MR imaging have been reported to be useful in subtyping meningiomas and intratumoral circulation. ${ }^{1,6-8)}$ The micro-vessel regions of meningiomas determined by histopathology were found to correlate with the relative cerebral blood volume (CBV) derived from perfusion MR images $^{6}$. However, the roles of CBV and CBF on perfusion MR imaging and diffusion signal intensity in evaluating preoperative histopathological and physiological information of meningiomas have not 
been fully examined to our knowledge. We analyzed the relationships between radiological findings on perfusion MR imaging and pathological characteristics such as origin of the tumor, mitotic activity, pathological subtype, and perifocal edema formation in meningiomas.

\section{Materials and Methods}

\section{Patients}

Inclusion criteria in this study were as follows: surgically treated meningioma at our institute, gross total or subtotal resection of the tumor within 10 days after MR examination and pathological confirmation. Meningiomas including intratumoral hemorrhage or cystic lesions in most components were excluded. We excluded meningiomas in which tumor invasion into the venous sinus and venous compression was suspected on MR images, and in which venous compression was directly observed during surgery. In all, 21 patients with newly diagnosed meningiomas with peritumoral edema were enrolled between April 2015 and March 2018. This study was approved by the Institutional Review Board of Sendai Medical Center, Sendai, Japan. Brain tumor data were extracted from an institutional database that includes consecutive patients with surgically treated meningiomas.

\section{MR protocol}

The protocol followed the methods reported by Zhang et al. ${ }^{8)}$ MR examinations were performed using a 1.5-Tesla clinical system (Signa; GE Medical Systems; Milwaukee, WI, USA). Three-plane localizer, sagittal and axial T1-weighted SE (TR/TE $=440 / 14 \mathrm{~ms}$; matrix, $256 \times 256$; section thickness, $8 \mathrm{~mm}$; field of view, $22 \mathrm{~cm}$ ) and axial fluid-attenuated inversion recovery (FLAIR, TR/TE $=8002 / 126 \mathrm{~ms}$, TI $=2,000 \mathrm{~ms}$ ) sequences were performed before contrast injection. Then, 15-20 mL of contrast agent (Gd-DTPA, 0.1 mmol/kg, Omniscan, GE Medical Systems) was administered intravenously using a power injector (Medrad, Indianola, PA, USA) at a rate of $4 \mathrm{~mL} / \mathrm{s}$, followed by a $20-\mathrm{mL}$ saline flush at the same rate. Five seconds after starting the injection, a $\mathrm{T} 2{ }^{*}$-weighted dynamic susceptibility perfusion series was started using a gradient-echo echo-planar sequence with the following parameters: TR/TE/excitations, 1800/40/1; flip angle, $60^{\circ}$; bandwidth, 62.75 ; matrix, $128 \times 128$; number of sections, 10; section thickness, $8 \mathrm{~mm}$ without spacing; field of view, $22 \mathrm{~cm}$; acquisition time, 1 min $31 \mathrm{~s}$. A series of 50 dynamic acquisitions was obtained for each section. Then, post-contrast T1-weighted sagittal and axial SE sequences were performed using the same section positions and parameters as in the pre-contrast series.

\section{Immunohistological analyses}

The MIB-1 labelling index (mean of positive nuclei/square millimeter) was calculated by recording the percentage of positively stained tumor cell nuclei out of 1000 tumor nuclei. Distinct nuclear staining was recorded as positive. Regions with the most immunostaining (known as hot spots) were used for calculating the labeling index. Measurement of intratumoral microvessel density was performed to evaluate the angiogenetic potential in meningiomas. This was measured by assessing immunohistochemical expression of CD34 in the most vascular areas (known as hot spots), as described by Weidner. ${ }^{9)}$ The area containing the maximum number of discrete microvessels was first identified and individual microvessels (mean of stained endothelial spaces/ square millimeter) were counted using a high-power objective. MIB-1 index and microvessel density were plotted compared with CBF, CBV and DW signal density, and correlations among them were analyzed.

\section{Data analysis}

Image analysis was performed using Functool software (ADW 4.0, GE Medical Systems) and the analytical protocol was carried out according to the method reported by Zhang et al. ${ }^{8)}$ A dynamic perfusion technique with contrast medium demonstrating cerebral hemodynamics by analyzing changes in signal intensities after the administration of contrast material was used in this study. ${ }^{10,11)}$ During the first phase of passage of the contrast agent bolus, both the $\mathrm{T} 2$ * relaxation time and its equivalent $\mathrm{T} 2$ * signal intensity decrease. The change in the relaxation rate $\left(\Delta \mathrm{R} 2^{*}\right)$ can be calculated based on the signal intensity by the following equation: (Rosen): $\Delta \mathrm{R} 2{ }^{*}=$ $\ln [S(t) / S 0] /$ echo time, in which $S(t)$ and So are the signal intensities at time $t$ and baseline. The recirculation and T1 shortening effects caused by contrast agent leakage can be reduced by fitting a gammavariate function to the measured $\Delta \mathrm{R} 2{ }^{*}$ curve. The area under the corrected contrast agent concentration-time curve is proportional to the CBV and does not yield an absolute measurement. Therefore, it is necessary to express the measurement relative to a standard reference, usually the contralateral white matter. We refer to this as the relative CBV. The steps to create perfusion maps were described in detail previously by Wang et al. ${ }^{7)}$ The source images were first inspected for overall image quality and motion artifacts. A single region of interest (ROI) was placed over the white matter of the contralateral unaffected centrum semiovale and CBV maps were generated to serve as a road map (Fig. 1). CBV maps were then calculated on a pixel-by-pixel basis and displayed as a grayscale image. However, small but 


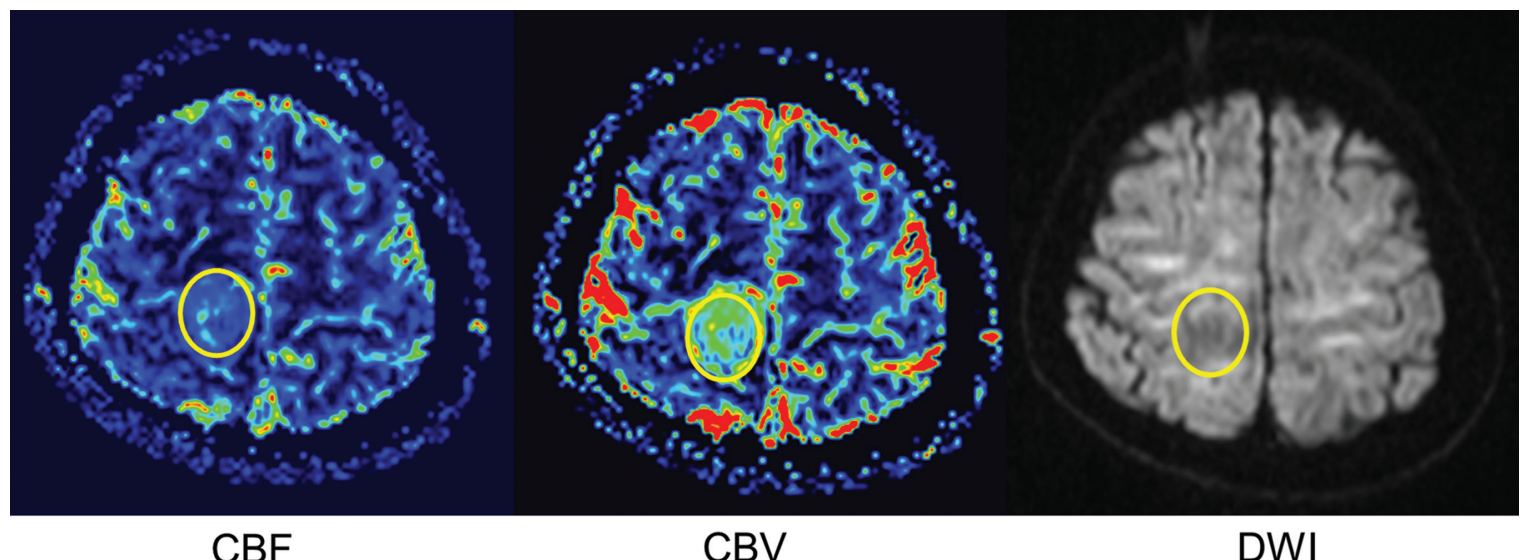

Fig. 1 MR images showing representative intratumoral circulatory findings for CBF and CBV, and signal intensity on a DW image of a right-falx meningioma. The yellow outline indicates the ROI on the meningioma. CBF: cerebral blood flow, CBV: cerebral blood volume, DW: diffusion-weighted, MR: magnetic resonance, ROI: region of interest.

important variations in CBV are not always apparent from these maps. An alternative is to use a color overlay displayed on the raw image in which the abnormal CBV values are often more apparent. Application of a threshold for the color overlay at a CBV the same as that of the unaffected white matter was used in this study. A color scale from dark (minimum CBV) to white (maximum CBV) is shown on the maps as reference. In lesions that are located close to major vascular structures, careful inspection of the images of different sequences revealed vessels that needed to be avoided. At least three circular ROIs, at least $20 \mathrm{~mm}^{2}$ each, were placed in the areas of the highest CBV of the solid part of the tumor parenchyma and peritumoral edema, and the maximal values were recorded (Fig. 1). Peritumoral edema in this study was defined as high signal on FLAIR images within a 1-cm distance from the outer enhanced tumor margin on post-contrast T1-weighted images. For semi-quantitative analysis, normal white matter within the contralateral hemisphere was used as the internal reference standard; CBV values were calculated by dividing the maximal CBV of a tumor by that of contralateral normal white matter. Statistical analysis was performed using a commercially available statistical software package (SPSS, version 14.0 for Windows; SPSS, Chicago, IL, USA). The mean CBF, CBV, and diffusion-weighted (DW) signal intensity of each group were expressed as the mean \pm standard deviation. These radiological data were compared with the tumor volume, relative ratio of perifocal edema, localization, and pathological malignancy.

The volume of perifocal edema was calculated according to the method reported by Sheehan et al. ${ }^{12)}$ The volume was defined as the high-signal intensity on T2-weighted images reflecting the peritumoral area subtracted from the volume of the enhanced area on T1-weighted images. The large peritumoral edema group comprised patients with a volume of peritumoral edema larger than that of the tumor itself. The non-large peritumoral edema group comprised patients with a volume of peritumoral edema less than that of the tumor itself. The ratio was calculated as the ROI data from the tumor side against that from the contralateral side according to the method by Zikou et al. ${ }^{13)}$ The differences between the two groups were compared using the Student's t-test. A p value less than 0.05 was considered significant. Pearson's product-moment correlation coefficient was used to measure association because most of the data had a normal distribution and the $r$ value was calculated as the correlation coefficient. Two-tailed $\mathrm{p}$ values were calculated and those greater than 0.05 were considered insignificant.

\section{Results}

\section{Clinical characteristics}

The subjects were 21 patients ( 7 men and 14 women) and the average age was 58.7 (32-85 years old). Clinical characteristics are shown in Table 1. The WHO grades were grade I in 17 , grade II in 3 , and grade III in 1. Pathological findings were meningothelial in eight, transitional in six, atypical in three, fibrous in two patients, microcystic in one, and anaplastic in one patient. The origin of tumors was the skull base in 11 and others in 10. Among tumors of skull base origin, five originated in the sphenoidal ridge, three in petrous bone, two in the middle fossa, and one in the olfactory groove. For the others, seven originated in the convexity, one 
Table 1 Clinical characteristics of 21 cases of meningioma

\begin{tabular}{lc}
\hline Cases & 21 (7 men, 14 women $)$ \\
\hline Age & Ave. 58.5 y.o. (32-85) \\
WHO grade & 3 \\
I & 17 \\
II & \\
III & 8 \\
Subtype & 6 \\
Meningothelial & 2 \\
Transitional & 1 \\
Fibrous & 3 \\
Microcystic & 1 \\
Atypical & \\
Anaplastic & 11 \\
Origin & 10 \\
Skull base & $48.4(4.2-131)$ \\
Non-skull base & $35(2.0-147)$ \\
Tumor volume $\left(\mathrm{cm}^{3}\right)$ &
\end{tabular}

Ave.; average, y.o.; years old

in the falx, one in the parasagittal area, and one in the intraventricle area.

\section{MR imaging findings}

The tumor volume was $4.2-131 \mathrm{~cm}^{3}$ (average was $48.4 \mathrm{~cm}^{3}$ ) and the volume of perifocal edema was 2-147 $\mathrm{cm}^{3}\left(35 \mathrm{~cm}^{3}\right)$ (Table 1). On perfusion MR imaging, the average ratio of CBV against the contralateral side was $6.43(1.13-20.0)$, that of CBF was 7.73 (1.34-28.3) and signal intensity on DW images was $1.27(0.80-2.74)$. There was no significant difference in CBF, CBV, or DW signal intensity among histological types.

Immunohistochemical analyses demonstrated the average MIB-1 index to be 5.4 and average microvessel density to be 58.9 microvessels/square millimeter. The MIB-1 index had no significant relationship with CBF, CBV, or DW signal intensity. There was no significant difference in MIB-1index among peritumoral edema or origin of the tumor. Microvessel density was not significantly correlated with CBF or DW signal density. There was no significant difference in microvessel density among peritumoral edema or origin of the tumor. Microvessel density was significantly correlated with CBV $(\mathrm{r}=0.61, \mathrm{p}=$ 0.03) (Fig. 2).

We defined the large peritumoral edema group and the non-large peritumoral edema group to examine the relationship between MR imaging data and peritumoral edema. The large peritumoral edema group comprised those with a volume of peritumoral edema larger than that of the tumor itself (edema > tumor) (Fig. 3). The non-large peritumoral edema group comprised those with a volume of peritumoral edema less than that of the tumor itself (edema < tumor) (Fig. 3). The large peritumoral edema group had a higher CBF and CBV, and lower DW signal intensity. The average CBF was 16.2 in the large peritumoral edema group and 12.2 in the non-large peritumoral group ( $\mathrm{p}<0.05)$ (Fig. 3). The average CBV was 7.5 in the large peritumoral group and 4.3 in the non-large peritumoral group $(\mathrm{p}<0.05)$ (Fig. 2). The average signal intensity on DW images was 1.03 in the large peritumoral group and 1.29 in the non-large peritumoral group $(\mathrm{p}=0.09)$ (Fig. 3).

We defined the skull base group and non-skull base group to assess tumor location. There was a significant difference in CBV and DW signal intensity between the two groups. Cerebral blood flow (CBF) had no significant relationship with tumor location (5.4 in the skull base group and 4.9 in the non-skull base group) ( $p=0.12$ ). The skull base group had a significantly higher CBV (7.6 in the skull base group and 4.2 in the non-skull base group) ( $\mathrm{p}<0.05$ ) and lower signal intensity on DW images than the non-skull base group (1.17 in the 


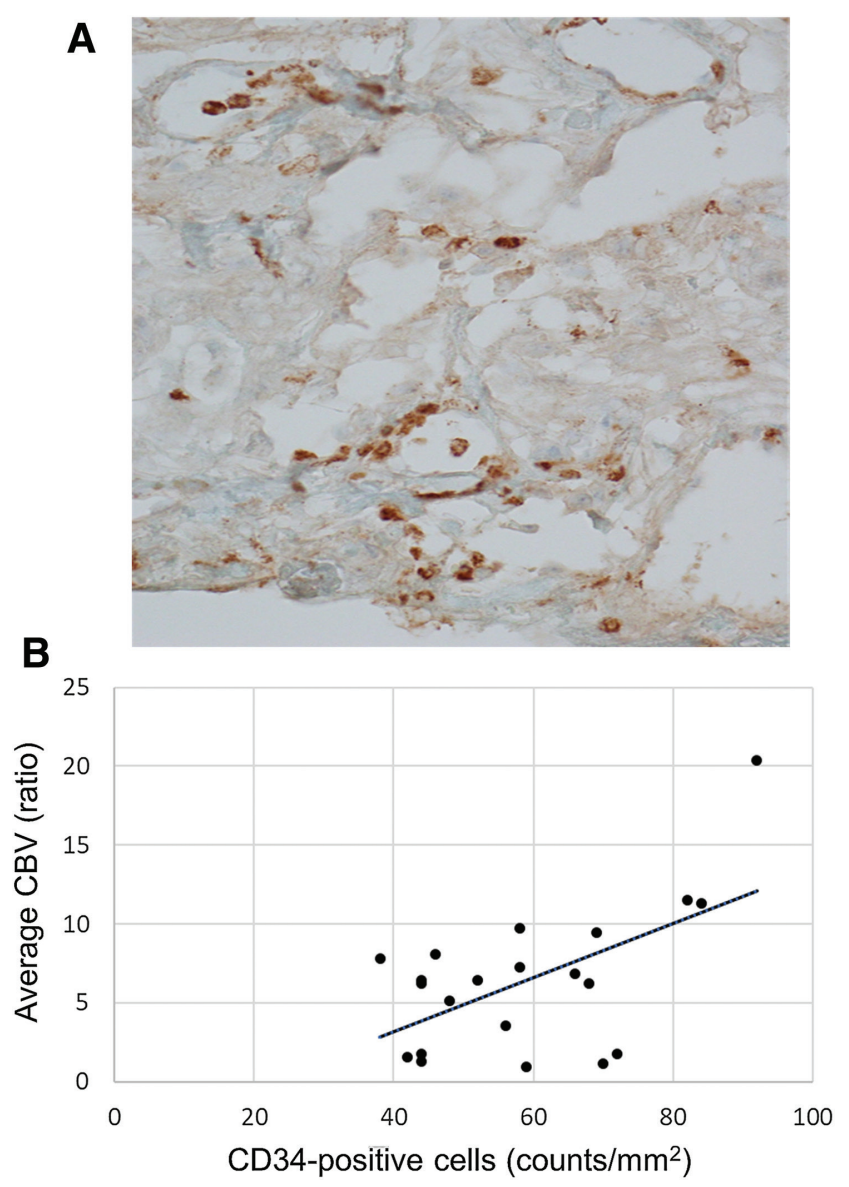

Fig. 2 Representative photograph showing immunohistological findings of CD34-positive cells in meningothelial-type meningioma $(\times 400)(A)$ Microvessel density was measured by assessing immunohistochemical expression of CD34 in the most vascular areas (mean of stained endothelial spaces/square millimeter). Graph showed correlation between average CBV (ratio) (longitudinal axis) and the number of CD34-positive cells (counts/millimeter) (horizontal axis). Microvessel density was significantly correlated with the average cerebral CBV ( $r=0.61, p=0.03$ ) (B). CBV: cerebral blood volume

skull base group and 1.39 in the non-skull base group) ( $\mathrm{p}<0.05$ ) (Fig. 4).

WHO grades and perfusion MR imaging data were evaluated. There was no significant difference in CBV or CBF between WHO grade I and grade II or III (CBV: 1.35 in grade I and 1.57 in grade II or III [p $=0.18$, CBF: 1.22 in grade $\mathrm{I}$ and 1.59 in grade II or III $[p=0.09])$. However, the average signal intensity on DW images was 1.52 in grade II or III and 1.19 in grade I ( $<0.05)$ (Fig. 4).

\section{Discussion}

Dynamic contrast-enhanced perfusion MR imaging studies demonstrated that relative CBV maps and

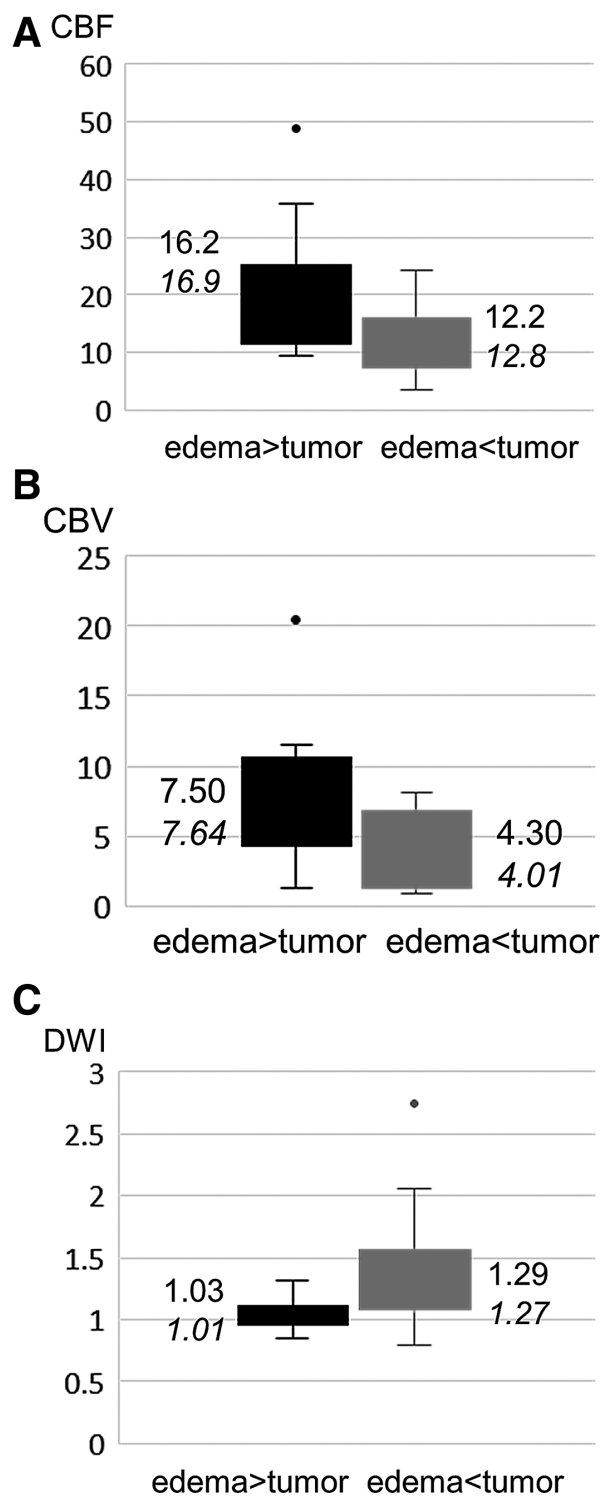

Fig. 3 Semi-quantitative analysis of the relationship between perfusion and DW MR images and peritumoral edema. The large peritumoral edema group comprised those with a volume of peritumoral edema larger than that of the tumor itself (edema > tumor). The non-large peritumoral edema group comprised those with a volume of peritumoral edema less than that of the tumor itself (edema < tumor). The average CBF ratio was higher in the edema $>$ tumor group (16.2) than in the edema < tumor group (12.2) (p <0.05) (A). The average $\mathrm{CBV}$ ratio was larger in the edema $>$ tumor group (7.5) than in the edema < tumor group (4.3) (p < 0.05) (B). The average signal intensity on DW images was lower in the edema $>$ tumor group (1.03) than in the edema $>$ tumor group $(1.29)(p<0.05)(C)$. Each median value was described under average value in italic typeface. CBF: cerebral blood flow; CBV: cerebral blood volume; DW: diffusion-weighted; MR: magnetic resonance 

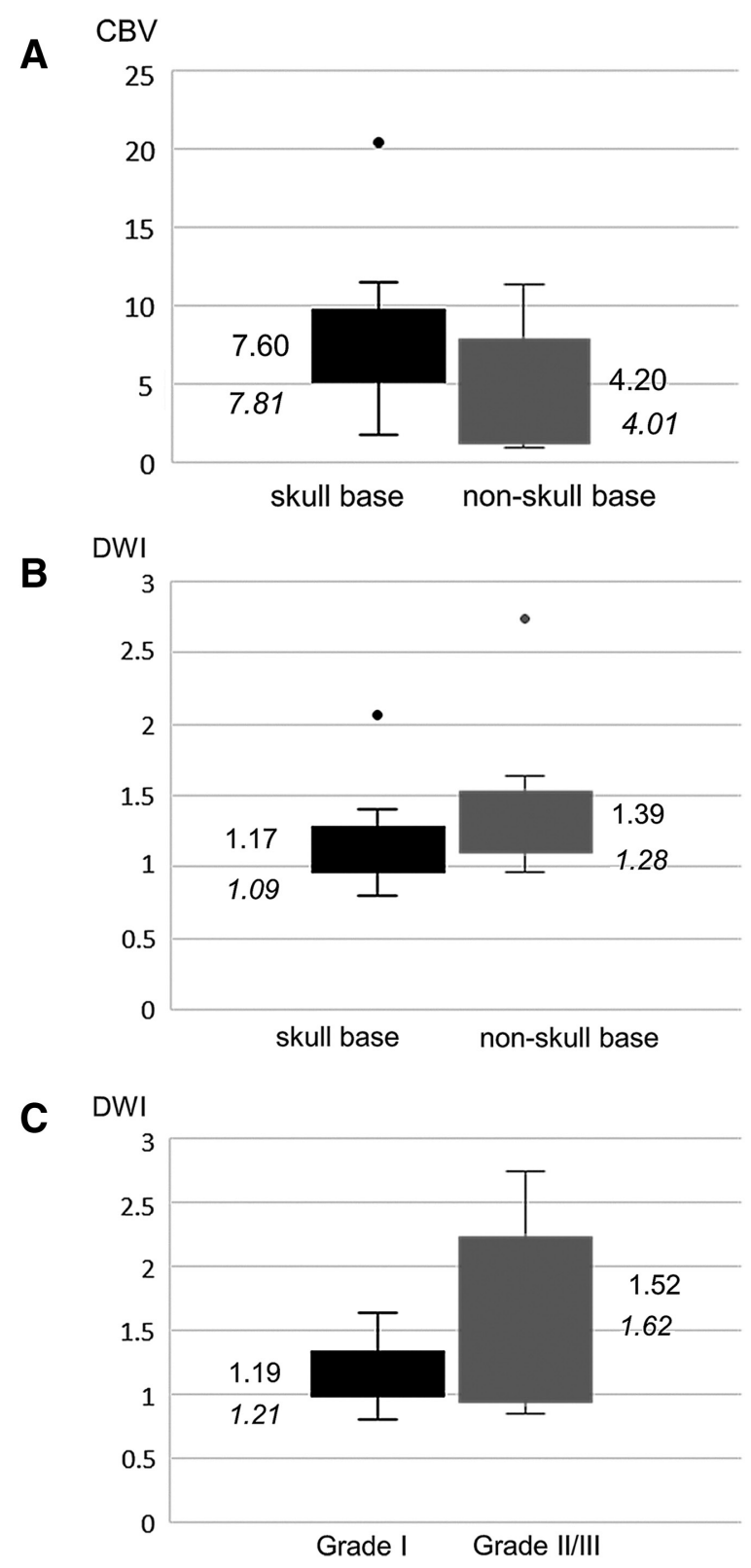

Fig. 4 Semi-quantitative analysis of the relationship between perfusion and DW MR images and tumor origin. Tumor origin was classified into two groups: originating from the skull base and non-skull base. The skull base group had a significantly higher CBV (7.6 in the skull base group and 4.2 in the non-skull base group) (p < 0.05) (A) and lower signal intensity on DW images than the non-skull base group (1.17 in the skull base group and 1.39 in the non-skull base group) (p <0.05) (B). Based on the WHO classification and perfusion MR imaging data, there was no significant difference in CBV or CBF (data not shown). However, the average signal intensity on DW images was 1.52 in grade II or III and 1.19 in grade $I(p<0.05)(C)$. Each median value was described under average value in italic typeface. DW: diffusion-weighted; MR: magnetic resonance
CBF maps can be used to grade gliomas, differentiate brain tumor types, and distinguish tumors from non-neoplastic lesions. ${ }^{14-16)}$ There have been several reports of preoperative MR imaging regarding the histopathological findings of meningiomas. ${ }^{2,3,17)}$ On conventional MR images of meningiomas, if the tumor is composed primarily of fibrous or transitional elements, it is significantly hypo-intense on T2-weighted MR images. ${ }^{17,18)}$ On the other hand, if the tumor is composed primarily of syncytial or angioblastic elements, it is significantly hyper-intense on T2-weighted MR images. Thus, hyper-intensity on T2-weighted images is associated with meningothelial, malignant, and angiomatous meningiomas, whereas hypointensity on T2-weighted images is associated with fibroblastic and transitional meningiomas. ${ }^{17,18)}$

On perfusion MR images of different meningiomas, Kimura et al. evaluated the regional CBV using continuous arterial spin labeling and dynamic susceptibility contrast perfusion MR imaging methods in a small group of patients. ${ }^{6)}$ They demonstrated that the CBV was largest in the angiomatous meningiomas, lowest in the fibrous-type meningiomas, and ranged from low to moderately high in meningothelial meningiomas. ${ }^{6}$ ) The mean CBV of angiomatous meningiomas was significantly different from that of the other two subtypes. ${ }^{6)}$ Zhang et al. ${ }^{8}$ reported a relationship between the pathological subtype and perfusion MR imaging findings of 37 meningiomas focusing on peritumoral edema. The maximal CBV in the tumoral parenchyma and peritumoral edema of each tumor was measured. The mean CBV in the tumoral parenchyma of angiomatous meningiomas and that in the peritumoral edema of anaplastic meningiomas were significantly different $(p<0.05)$ from those of the other types of meningiomas.

To our knowledge, the following are novel findings in the present study: First, meningiomas with large perifocal edema often had a higher CBF and CBV. Second, meningiomas originating from the skull base often had a higher CBV and lower signal intensity on DW images. Third, WHO grade II or III meningiomas often had a higher signal intensity on DW images. Fourth, meningiomas with high microvessel density had a higher CBV. In our study, we set the ROI in the tumor parenchyma and compared the data with the contralateral white matter according to the method reported by Zhang et al. ${ }^{8)}$ The large peritumoral edema group was defined as those with a larger hyper-intense volume than the tumor volume on T2-weighted images. The extent of tumor volume did not affect the CBV, CBF, or DW signal intensity, but large peritumoral edema caused a higher CBF and CBV. This suggests higher vascularity inside of the tumors, which may increase 
the permeability of the parenchymal blood barrier and transportation of water. Progression of perifocal edema is caused by numerous mechanisms. Further studies with perfusion MR findings may help predict the future progression of perifocal edema. The origin of the tumor was evaluated as skull base or non-skull base in our study. Meningiomas originating from the skull base had a lower signal intensity on DW images, suggesting lower cellular intensity. Microvessel density was focused on as a factor of microcirculatory status of the meningioma and was correlated only with the intratumoral CBV. Microvessel formation is considered a factor for formation of the intratumoral vascular bed. Perifocal edema formation and tumor location may be mutually involved in intratumoral vascular bed formation with circulatory factors such as arterial blood supply, venous circulation, and tumor cellular density.

Mansouri et al. ${ }^{19)}$ reported a correlation between cellular growth and the localization of meningiomas, with significantly lower cellular growth in the skull base meningiomas. Our DW signal intensity data may reflect the cellular growth of skull base meningiomas. Similar to the correlation of DW signal intensity with WHO grades, higher-grade tumors exhibited higher signal intensity on DW images. Higher intratumoral blood volume may reflect hyper-vascularity and development of a vascular bed such as angiomatous meningiomas. ${ }^{20)}$ On the other hand, high blood flow inside of meningiomas may cause a larger amount of venous drainage and arterial venous shunts, resulting in higher permeability and higher peri-tumoral edema formation. The precise mechanism of high tumoral blood flow was unable to be examined in this study. The retrospective nature, manual setting of ROIs, and the small number of subjects in our study were limitations. Another limitation was the evaluation of edema formation caused by venous compression in this study. We excluded patients in which tumor invasion into the venous sinus and venous compression caused by the tumor were observed on MR images or during surgery. However, destruction of venous circulation in small veins surrounding the tumor may not be fully excluded.

Preoperative perfusion MR images of meningiomas may provide useful information to predict peritumoral edema formation, tumor growth, and cellular malignancy transformation in the future. Further prospective studies may be required.

\section{Conclusion}

Perfusion MR imaging revealed that a higher ratio of peritumoral edema to tumor size reflects higher blood flow and blood volume under intratumoral circulatory conditions, and that skull base meningiomas have a higher blood volume than non-skull base meningiomas. Our study may provide useful information to predict peritumoral edema formation, tumor growth, and cellular malignancy in the future.

\section{Acknowledgment}

We would like to thank our medical staff and radiologists for data acquisition.

\section{Conflicts of Interest Disclosure}

All authors declare that they have no conflicts of interest concerning the material or methods used in this study, or the findings presented in this manuscript. No financial or material support was received. The content of this article has not been published or presented previously.

\section{References}

1) Toh CH, Wei KC, Chang CN, et al.: Assessment of angiographic vascularity of meningiomas with dynamic susceptibility contrast-enhanced perfusion-weighted imaging and diffusion tensor imaging. AJNR Am J Neuroradiol 35: 263-269, 2014

2) Wiemels J, Wrensch M, Claus EB: Epidemiology and etiology of meningioma. J Neurooncol 99: 307-314, 2010

3) Sasao A, Hirai T, Nishimura S, et al.: Assessment of vascular supply of hypervascular extra-axial brain tumors with $3 \mathrm{~T}$ MR regional perfusion imaging. AJNR Am J Neuroradiol 31: 554-558, 2010

4) Villringer A, Rosen BR, Belliveau JW, et al.: Dynamic imaging with lanthanide chelates in normal brain: contrast due to magnetic susceptibility effects. Magn Reson Med 6: 164-174, 1988

5) Warach S, Dashe JF, Edelman RR: Clinical outcome in ischemic stroke predicted by early diffusion-weighted and perfusion magnetic resonance imaging: a preliminary analysis. J Cereb Blood Flow Metab 16: 53-59, 1996

6) Kimura H, Takeuchi H, Koshimoto Y, et al.: Perfusion imaging of meningioma by using continuous arterial spin-labeling: comparison with dynamic susceptibility-weighted contrast-enhanced MR images and histopathologic features. AJNR Am J Neuroradiol 27: 85-93, 2006

7) Wang S, Kim S, Zhang Y, et al.: Determination of grade and subtype of meningiomas by using histogram analysis of diffusion-tensor imaging metrics. Radiology 262: 584-592, 2012

8) Zhang H, Rödiger LA, Shen T, Miao J, Oudkerk M: Perfusion MR imaging for differentiation of benign and malignant meningiomas. Neuroradiology 50: 525-530, 2008 
9) Weidner N: Tumour vascularity and proliferation: clear evidence of a close relationship. J Pathol 189: 297-299, 1999

10) Rosen BR, Belliveau JW, Vevea JM, Brady TJ: Perfusion imaging with NMR contrast agents. Magn Reson Med 14: 249-265, 1990

11) Weisskoff R, Belliveau J, Kwong K: Functional MR imaging of capillary hemodynamics. In: Potchen E (eds) Magnetic resonance angiography: concepts and applications. pp 473-484, St Louis, Mosby, 17, 1993

12) Sheehan JP, Lee CC, Xu Z, Przybylowski CJ, Melmer PD, Schlesinger D: Edema following Gamma Knife radiosurgery for parasagittal and parafalcine meningiomas. J Neurosurg 123: 1287-1293, 2015

13) Zikou AK, Xydis VG, Astrakas LG, et al.: Diffusion tensor imaging in children with tuberous sclerosis complex: tract-based spatial statistics assessment of brain microstructural changes. Pediatr Radiol 46: 1158-1164, 2016

14) Cha S: Perfusion MR imaging: basic principles and clinical applications. Magn Reson Imaging Clin N Am 11: 403-413, 2003

15) Hakyemez B, Erdogan C, Bolca N, Yildirim N, Gokalp G, Parlak M: Evaluation of different cerebral mass lesions by perfusion-weighted MR imaging. J Magn Reson Imaging 24: 817-824, 2006

16) Kremer S, Grand S, Remy C, et al.: Cerebral blood volume mapping by MR imaging in the initial evaluation of brain tumors. J Neuroradiol 29: 105-113, 2002

17) Ildan F, Tuna M, Göçer AP, et al.: Correlation of the relationships of brain-tumor interfaces, magnetic resonance imaging, and angiographic findings to predict cleavage of meningiomas. J Neurosurg 91: 384-390, 1999

18) Chen TC, Zee CS, Miller CA, et al.: Magnetic resonance imaging and pathological correlates of meningiomas. Neurosurgery 31: 1015-1021; discussion 1021-1022, 1992

19) Mansouri A, Klironomos G, Taslimi S, et al.: Surgically resected skull base meningiomas demonstrate a divergent postoperative recurrence pattern compared with non-skull base meningiomas. J Neurosurg 125: 431-440, 2016

20) Koizumi S, Sakai N, Kawaji H, et al.: Pseudo-continuous arterial spin labeling reflects vascular density and differentiates angiomatous meningiomas from non-angiomatous meningiomas. J Neurooncol 121: 549-556, 2015

Corresponding author: Atsushi Saito, MD, PhD Department of Neurosurgery, Kohnan Hospital, 4-20-1 Nagamachiminami, Taihaku-ku, Sendai, Miyagi 982-8523, Japan. e-mail: satsushi2002@yahoo.co.jp 